\title{
Case Report \\ A case of colonic carcinoma presenting as acquired haemophilia A
}

\author{
Arthihai Srirangan $^{1}$, Jegarajah indrakumar ${ }^{2}$ \\ ${ }^{1}$ Colombo South Teaching Hospital, ${ }^{2}$ Faculty of Medical Sciences, University of Sri Jayewardenepura, \\ Sri Lanka
}

\section{Keywords: Acquired Haemophilia A, Factor VIII inhibitor, Colonic Carcinoma}

\author{
Corresponding Author: Arthihai Srirangan, E-mail: < arthi1607@yahoo.com > (D) https://orcid.org/0000-0002-4905-6393 \\ Submitted: November 2019, Accepted: June 2020, Published June 2020 \\ Competing Interests: Authors have declared that no competing interests exist \\ (C) Authors. This is an open-access article distributed under a Creative Commons Attribution-Share \\ Alike 4.0 International License (CC BY-SA 4.0), which permits unrestricted use, distribution, and \\ reproduction in any medium, provided the original author and source are attributed and materials \\ are shared under the same license.
}

\section{Introduction}

Acquired haemophilia $\mathrm{A}(\mathrm{AHA})$ is a rare cause of severe life-threatening bleeding in the elderly. It is characterised by autoantibodies against circulating coagulation factor VIII (FVIII) which impair the intrinsic coagulation system [1]. The diagnosis of AHA should be considered in an elderly patient presenting with spontaneous bleeding and isolated prolongation of the activated partial thromboplastin time (aPPT). Prompt diagnosis, treatment to control severe bleeding and suppression of inhibitors by immunosuppressive treatment is lifesaving. Up to half of the cases are idiopathic. It has a bimodal distribution, with the first peak in women in the postpartum period and the second among elderly patients, in whom it is commonly linked with autoimmune disease, solid tumours and drug reactions [2]. In an elderly patient with $\mathrm{AHA}$, an underlying disease pathology should always be considered, as the treatment of the coexisting disease will eradicate the inhibitors.

We report a patient who initially presented with AHA and was subsequently detected to have a colonic carcinoma.

\section{Case presentation}

A 70-year-old, previously healthy male presented with a 4 days history of left upper arm swelling and erythema which was initially managed as left upper-limb cellulitis. However, the condition progressed despite antibiotic treatment and he developed generalized extensive ecchymotic patches involving the upper chest and lower limb. There were no other bleeding manifestations, such as haematuria, gastrointestinal bleeding, haemoptysis or epistaxis. He denied any personal or family history of a bleeding diatheses. There were no clinical features to suggest an underlying autoimmune disorder.

On clinical examination, he was haemodynamically stable, yet severely paled. He had multiple ecchymotic patches over the chest, upper limbs and thighs with prominent left arm swelling and erythema. There was no evidence of haemarthrosis. His cardiovascular, respiratory and abdominal examination was essentially normal.

Laboratory investigation on admission revealed a haemoglobin of $6 \mathrm{~g} / \mathrm{dl}$, a platelet count of $233 \times 10^{9} / \mathrm{L}$ and a white blood cell count of $12 \times 10^{\%} / \mathrm{L}$. The coagulation 
profile showed a prolonged aPPT of 65 seconds (30-40s) with a normal PT/INR and bleeding time. Mixing studies were performed; however, aPPT failed to correct with normal plasma. This supported the presence of a specific coagulation factor inhibitor rather than a deficiency. Plasma factor VIII activity (FVIII:C) was found to be as low as 32iu/dL (normal 50-150) and the FVIII inhibitor was 5.6 Bethesda units $/ \mathrm{ml}$ (reference range $\leq 0.50$ ). Other clotting factors were normal. A diagnosis of acquired haemophilia $A$ was made.

He was symptomatically treated with a transfusion of 3 units of packed red cells to correct the anaemia. Haemostasis was achieved with Factor VIII inhibitor bypassing activity (FEIBA) $50 \mathrm{U} / \mathrm{kg} /$ day, which was given for two days to bypass the activity of the inhibitory autoantibody. He was given immunosuppressive therapy in the form of high dose prednisolone $1 \mathrm{mg} / \mathrm{kg} /$ day $(60 \mathrm{mg})$ with tailing off and cyclophosphamide $2 \mathrm{mg} / \mathrm{kg} /$ day $(100 \mathrm{mg})$. The patient was discharged after 2 weeks with an aPTT of $45 \mathrm{~s}$ and with resolution of the ecchymotic patches. There were no new haemorrhagic manifestations during the hospital stay.

His aPPT was corrected on subsequent clinic follow up. He had undergone upper and lower gastro endoscopy which were normal. Contrast-enhanced CT (CECT) of the chest, abdomen, and pelvis revealed no abnormalities. His autoimmune screening was also negative.

Eight months later, while on clinic follow up and treatment, the patient developed severe loss of appetite, loss of weight, and a cough. Examination revealed left cervical lymphadenopathy with bilateral axillary lymphadenopathy. Chest radiograph revealed cannonball lesions. The CECT abdomen demonstrated a thickened enhancing segment at the splenic flexure with multiple liver and lung metastases and mediastinal lymph nodes. Left axillary lymph node histology revealed a metastatic adenocarcinoma. The patient was diagnosed to have metastatic colonic carcinoma and transferred to the cancer hospital for palliative treatment.

\section{Discussion}

AHA is a potentially serious bleeding disorder with significant mortality and morbidity. As indicated by PW Collins, the estimated annual prevalence is $1.48 /$ million per year with over $80 \%$ being over the age of sixty years [3]. It is an acquired autoimmune disease characterized by an autoantibody against FVIII which inactivates it and increase its clearance from the blood. Unlike hereditary haemophilias in which bleeding occurs into joints, AHA typically presents as the sudden appearance of extensive cutaneous purpura, as found in our patient, or internal haemorrhage, both of which are potentially fatal [3].

AHA must be considered in bleeding patients with isolated, prolonged aPTT with a normal PT. The mixing test is a basic assay able to reveal the presence of inhibitors of coagulation, in which the plasma of the patient is mixed with normal plasma in a proportion of $1: 1$. The patient's inhibitors of FVIII will also inhibit the FVIII in the normal plasma. Subsequently, quantitative coagulation factor assays should be performed to reveal a reduced level of FVIII: C in AHA. The diagnosis is then confirmed by a positive Bethesda assay [4].

The main aim in the treatment of patients with AHA is to control bleeding episodes, initiate immunosuppression to eradicate inhibitors and treat the underlying cause, 
if any [5]. The first-line treatment to control bleeding in AHA is the use of a bypassing agent, either recombinant factor VIIa, activated prothrombin complex concentrate (aPCC) or FEIBA. To eradicate the inhibitor, corticosteroids alone (prednisone 1-2 $\mathrm{mg} / \mathrm{kg} / \mathrm{day}$ for 4-6 weeks) or in combination with cyclophosphamide (1-2 $\mathrm{mg} / \mathrm{kg}$ per day for a maximum of 5 weeks) is recommended. Rituximab is now available as a second-line drug for refractory cases [6]. Therapeutic response is measured by evaluating the FVIII level which should return to normal and inhibitor titres which should become undetectable.

It has been reported that $10 \%$ of patients with AHA have an underlying malignancy [7]. It is unclear whether autoantibody development derives from the tumour itself or whether cancer antigens share immunological cross-reactivity with FVIII. AHA may be detected prior to the diagnosis of malignancy, as in our patient [8]. Since the patient had an advanced malignancy by the time he was diagnosed 8 months after the AHA diagnosis, it is very likely that the colonic carcinoma developed around the same time that he was initially diagnosed with AHA. Hence, it is important to follow up and exclude malignancies in these patients.

\section{Conclusions}

AHA should be considered in any elderly patient who presents with bleeding and an isolated prolonged aPTT. The mixing test can be easily carried out in all laboratories and will permit the identification of patients who require further evaluation. Undetected malignancy can initially present as AHA. Therefore, the physician needs to evaluate every case with close monitoring to detect an underlying malignancy.

\section{List of abbreviations \\ AHA acquired haemophilia $A$ \\ FVIII factor VIII \\ aPPT activated partial thromboplastin time \\ CECT Contrast-enhanced CT \\ FEIBA Factor VIII Inhibitor Bypassing Activity}

\section{Acknowledgements}

We would like to acknowledge the contribution of Dr. Nishadhya Ranasinghe, Consultant Haematologist, Colombo South Teaching Hospital for her support.

\section{References}

1. Kessler CM, Knobl P. Acquired haemophilia: An overview for clinical practice. Eur J Haematol. 2015;95(Suppl. 81):36-44. https://doi.org/10.1111/ejh.12689

2. Collins PW, Hirsch S, Baglin TP, et al. Acquired hemophilia A in the United Kingdom: A 2-year national surveillance study by the United Kingdom Haemophilia Centre Doctors' Organisation. Blood. 2007;109(5):1870-77. https://doi.org/10.1182/blood-2006-06-029850

3. Baudo F, Caimi T, De Cataldo F. Diagnosis and treatment of acquired haemophilia. Haemophilia 2010;16:102-6. https://doi.org/10.1111/j.13652516.2010.02232.x

4. Collins $P$, Baudo $F$, Huth-Kuhne $A$, et al. Consensus recommendations for the diagnosis and treatment of acquired hemophilia A. BMC Res Notes. 
$2010 ; 3: 161$.

https://doi.org/10.1186/1756-0500-3-161

5. Delgado J, Jimenez-Yuste V, Hernandez-Navarro F, Villar A. Acquired haemophilia: review and meta-analysis focused on therapy and prognostic factors. British journal of haematology. 2003 Apr;121(1):21-35. https://doi.org/10.1046/j.1365-2141.2003.04162.x

6. Franchini M, Vaglio S, Marano G, et al. Acquired hemophilia A: A review of recent data and new therapeutic options. Hematology. 2017;22(9):514-20. https://doi.org/10.1080/10245332.2017.1319115

7. Knoebl P, Marco P, Baudo F, et al. Demographic and clinical data in acquired hemophilia A: results from the European Acquired Haemophilia Registry (EACH2) J Thromb Haemost. 2012;10(4):622-31. https://doi.org/10.1111/j.1538-7836.2012.04654.x

8. Sakurai Y, Takeda T. Acquired hemophilia A: A frequently overlooked autoimmune hemorrhagic disorder. J Immunol Res. 2014;2014:320674.

https://doi.org/10.1155/2014/320674 